Gonet Klara, Zaborek Monika, Lyczba Jakub, Borowik Joanna, Przysucha Anna. Dose of information about laryngeal cancer with particular emphasis on laryngectomy. Journal of Education, Health and Sport. 2020;10(5):81-88. eISSN 2391-8306. DOI http://dx.doi.org/10.12775/JEHS.2020.10.05.007

https://apcz.umk.pl/czasopisma/index.php/JEHS/article/view/JEHS.2020.10.05.007

https://zenodo.org/record/3825963

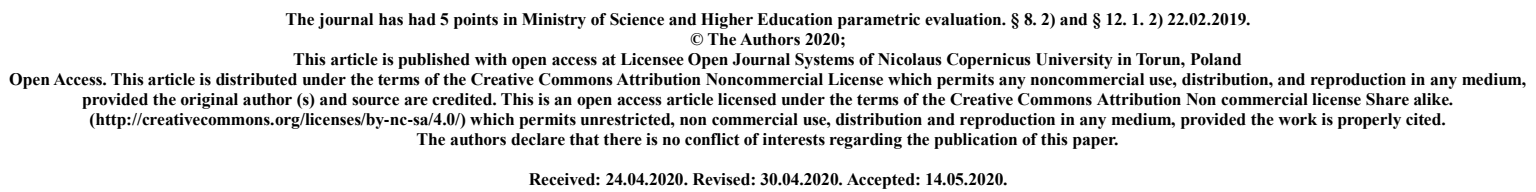

\title{
Dose of information about laryngeal cancer with particular emphasis on laryngectomy
}

\section{Klara Elżbieta Gonet, Monika Karolina Zaborek, Jakub Lyczba, Joanna Urszula Borowik, Anna Przysucha}

\footnotetext{
Name

Klara Elżbieta Gonet

Affiliation

Student's Scientific Group of Otolaryngology, Medical University of Lublin, Jaczewskiego Street 8, 20-954 Lublin

Country

Poland

Bio Statement

Principal contact for editorial correspondence.

Name

Affiliation

Country

Bio Statement

Name

Affiliation

Country

Bio Statement

Monika Karolina Zaborek

Student's Scientific Group of Otolaryngology, Medical University of Lublin

Poland

$$
\text { Jakub Łyczba }
$$

Student's Scientific Group of Otolaryngology, Medical University of Lublin

Poland
} 


$\begin{array}{ll}\text { Name } & \text { Joanna Urszula Borowik } \\ \text { Affiliation } & \text { Student's Scientific Group of Otolaryngology, Medical University of Lublin } \\ \text { Country } & \text { Poland } \\ \text { Bio Statement } & - \\ \text { Name } & \text { Anna Przysucha } \\ \text { Affiliation } & \text { Student's Scientific Group of Otolaryngology, Medical University of Lublin } \\ \text { Country } & \text { Poland } \\ \text { Bio Statement } & -\end{array}$

\begin{abstract}
Introduction and purpose:

The laryngeal cancer is the most common cancer of head and neck and is associated with smoking. It is mainly squamous cell carcinoma. There are many methods to diagnosis this cancer. The most important method of the treatment is complicated operation - laryngectomy which is described in this article. There are presented diagnosis and staging of laryngeal cancer, the main types of laryngectomy, process, results and complications. And the end of this article will be mentioned about the loss of voice and of normal and efficient verbal communication as a negative consequence associated with this type of surgery that can have significant impacts on the quality of life. The purpose of this article is to familiarize readers with the topic of laryngectomy as the primary treatment for larynx cancer.

Description of the state of knowledge:

Studies show an increase in the incidence of this type of cancer. The main risk factors are smoking, drinking alcohol and exposure to heavy metals. Symptoms are really different and include hoarseness, sore throat, coughing and dysphagia. Depending on the location, the course of the cancer can be different. The main examinations are laryngoscopy, CT, MRI, USG and PET. The laryngectomy procedure as a main therapy must be radical as far as possible to the extent that the tumor can be resected within healthy tissue but the goal is to preserve the largest possible function of the organ. The most often complication is pharyngocutaneous fistula.

Summary:

The laryngectomy is the main method of therapy of the laryngeal cancer. it is a mutilating and often speech-depriving procedure but medicine has now many solutions for this kind of patients.
\end{abstract}

Key words: laryngeal cancer; laryngectomy; loss of voice 


\section{Introduction and purpose}

The laryngeal cancer accounts for $2-4 \%$ of all cancers and is the most common cancer of head and neck. It is more common in men and is associated with smoking. It has many different symptoms depending on the localization. To diagnose we can use many examinations like direct and indirect laryngoscopy, videolaryngoscopy, CT, MRI, PET, USG and X-ray of the chest (to exclude metastasis). This cancer has a complicated staging and according to this, the ENT surgeons make a decision about treatment. The main method of the treatment is surgerylaryngectomia (partial or total) types and uses of which, extent and complications will be presented in this article. The purpose of this article is to familiarize readers with the topic of laryngectomy as the primary treatment for larynx cancer.

\section{Laryngeal cancer}

\subsection{Something about larynx}

The larynx is an organ which is located in the top of the neck at the level of the C3/C4-C6/C7 vertebrae and connects pharynx with the trachea. It consists of cartilages that are attached to each other and to the surrounding muscles. The larynx contains three cavities: epiglottis, glottis and subglottis. The mucous membrane lining the larynx forms two pairs of lateral folds: the two upper folds are called the vestibular folds and the two lower - vocal folds which produce sounds needed to speak. (1) The main functions of this organ are creating voice, conduit for air to enter lungs and protect the lungs from food, liquids and foreign bodies by coughing and other reflexive actions. Unfortunately some people have their larynx to be removed because in this organ is a developing cancer.

\subsection{Characteristics of larynx cancer}

The laryngeal cancer is the most common cancer of head and neck. $90 \%$ of them is squamous cell carcinoma. The incidence shows a moderate upward trend and men are seven times more likely to get ill. The main risk factors are smoking, drinking alcohol and exposure to heavy metals (2,3). Symptoms are really different and include hoarseness, sore throat, cough, dysphagia or odynophagia (trouble or pain when swallowing), ear pain, foreign body feeling, dyspnoea and hemoptysis. These symptoms can be caused by tumor or by other conditions $(2,4)$. Depending on the location, the course of the cancer can be different. Epiglottis cancer is characterized by rapid local growth, early metastasis in lymph nodes and rarely distant metastasis. Glottis cancer is characterized by slow local growth and rarely metastasis in lymph nodes. The third cancer - subglottis cancer - is the rarest $(10 \%)$ and has moderatly dynamic local growth but gives more often metastasis to lymph nodes not only in the neck but even to thoracic lymph nodes (3). All of them can spread through the blood, the lymph system and tissue (4).

2.3. Examinations that can help to make a diagnosis

Indirect laryngoscopy - this procedure allows visualization of the larynx using a mirror. We can see for example epiglottis, epiglottic tubercle, vestibular folds, vocal folds, aryepiglottic folds and entrance to the esophagus. The larynx is examined in two positions: respiratio vocal and vestibular folds are opened and fonatio - the folds are closed. Thanks to this we can observe the movement of the vocal folds which every time should be symmetrical.

Direct laryngoscopy - this procedure allows visualization of the larynx using a laryngoscope (a laryngoscope is a thin, tube-like instrument with a light and a lens for viewing the inside of 
human body). It is used during anesthesia and surgical procedures around the larynx. The variety is microlaryngoscope which allows you to accurately assess the location and size of the tumor. This study provides the most information on the location and severity of the tumor. You can accurately assess the tumor surface and accurately take a slice. Microlaryngoscopy can be both diagnostic and therapeutic. For diagnostic purposes, specimens for histopathological examination are taken, it is also used in intra-laryngeal microsurgery various pathological changes from the larynx are removed, e.g. polyps.

Videolaryngoscopy - this procedure allows recording an exam.

CT scan - every patient with laryngeal cancer should have CT of neck to asses the mass and exact location of the tumor, the state of the lymph nodes and surrounding organs. Assessment of the thyroid cartilage of the larynx is particularly important due to the fact that its extensive involvement and destruction eliminates the possibility of radiation-based conservative therapy of the larynx (3).

MRI - additional examination which allows to show soft tissue and define TNM.

USG - USG of the neck performs an auxiliary role in assessing the condition of the lymph nodes and also allows the biopsy of suspicious nodes (3).

PET - is used to find malignant tumor cells in the body. A small amount of radioactive glucose (sugar) is injected into a vein. The PET scanner rotates around the body and makes a picture of where glucose is being used in the body. Malignant tumor cells show up brighter in the picture because they are more active and take up more glucose than normal cells do (4).

PET-CT - is a procedure that combines the pictures from a positron emission tomography scan and a computed tomography scan. The PET and CT scans are done at the same time with the same machine (4).

X-ray of the chest - obligatory for every patient to exclude metastasis and another cancer (3).

\subsection{Stages of laryngeal cancer (TNM)}

The TNM staging system is the most common way of staging cancer of the larynx. It includes $\mathrm{T}$ - tumor, $\mathrm{N}$ - nodes and $\mathrm{M}$ - metastasis.

Tumor describes how far the cancer has grown. There are five T stages:

$\checkmark$ Tis (tumor in situ) - the disease is very early and has the best prognosis. It is contained in covering of the larynx (mucosa). It has not spread into surrounding tissue

$\checkmark \mathbf{T 1}$ - the tumour is only in one part of the larynx and the vocal cords are able to move normally

$\checkmark \quad \mathbf{T} 2$ - the tumour has grown into another part of the larynx

$\checkmark$ T3 - the tumour is more bulky and has caused one of the vocal cords to not move or the tumour has grown into nearby areas such as the tissue in front of the epiglottis or the inner part of the thyroid cartilage

$\checkmark$ T4 - the tumour has grown into body tissues outside the larynx

Node describes whether the cancer had spread to the lymph nodes. There are four $\mathrm{N}$ stages:

$\checkmark$ No - the lymph nodes don't contain cancer cells

$\checkmark \mathbf{N 1}$ - one lymph node contains cancer cells on the same side as the cancer and the node is less than $3 \mathrm{~cm}$ across

$\checkmark$ N2a - one lymph node contains cancer cells on the same side as the cancer and the node is between $3 \mathrm{~cm}$ and $6 \mathrm{~cm}$ across 
$\checkmark$ N2b - more than one lymph node contain cancer cells on the same side as the cancer but none of them are more than $6 \mathrm{~cm}$ across

$\checkmark$ N2c - cancer cells in lymph nodes on the other side or in nodes on both sides of the neck but none of the nodes are more than $6 \mathrm{~cm}$ across

$\checkmark \mathbf{N 3}$ - one lymph node containing cancer cells is larger than $6 \mathrm{~cm}$ across

Metastasis describes whether the cancer has spread to a different part of the body. There are two M stages:

$\checkmark$ M0 - no cancer spread

$\checkmark$ M1 - the cancer has spread to other parts of the body

The stage of the cancer helps to decide which treatment is the best for the patient (5).

2.5. Treatment with particular emphasis on laryngectomy

Untreated cancer leads to death usually within 12 months as a result of suffocation, infection, bleeding and metastasis. Coexistence of severe diseases significantly worsens the prognosis (2). Based on the specificity of this cancer, the main method of the treatment is surgical, because many years of experience show the ineffectiveness of chemotherapy and less effect than expected of radiation therapy (6). Radiotherapy found application mainly in case of T1 and T2, N0 (also $\mathrm{CO}_{2}$ endoscopic laser or larynx conserving treatment). Other indications are inoperable tumor, palliative disease and in the absence of agreement for surgery. Relative indication for radiation therapy is the involvement of the lower part of the throat. In selected patients (T3 and T4), the combination of surgery and postoperative radiotherapy brought better results. Radiotherapy complications include: swelling, dysphagia, dry mouth and lack of taste.

\subsubsection{Laryngectomy}

Laryngectomy is the surgical removal of some or all of the larynx. It is the basis for treating laryngeal cancer performed by the ENT surgeons. The first total laryngectomy was performed in 1873 by Theodor Billroth. The patient was a 36 year old man with a subglottic carcinoma. At first the laryngectomy was performed and after a few months recurrence was detected and the patient died (7).

The decision about type of laryngectomy must be taken very carefully. On the one hand, the procedure must be radical (as far as possible) to the extent that the tumor can be resected within healthy tissue, on the other hand, the goal is to preserve the largest possible function of the organ. However, the most important criterion for choosing is the extent of the tumor infiltration. Careful diagnosis determines its extent (we can use the above methods). it is important to take into account the age and condition of the patient and associated diseases. There are two types of laryngectomy: partial laryngectomy and total laryngectomy. 


\subsubsection{Partial laryngectomy}

In this operation only a portion of the larynx is removed. It can be done endoscopically or from an external approach. Partial laryngectomies are sometimes called functional, because removing a part of the larynx allows its function (respiratory, phonative and protective).

endoscopic chordectomy - endoscopic procedure through the mouth, performed under general anesthesia - laryngeal microsurgery. It involves the removal of the vocal fold.

Application: in the early forms of glottis cancer (pre-invasive cancer, microinvasive cancer), which are located in the middle part of the vocal fold.

$>$ extra-laryngeal chordectomy - performed from an external access.

Application: voice fold cancer located near the arytenoid cartilage. Opening the larynx from the outside gives a better insight and assessment of the extent of glottis cancer, and most importantly, such a removal of the lesion that ensures oncological purity.

$>$ partial frontal laryngectomy - performed by the Leroux-Robert method or by the Tapia method. It consists in removing the anterior sections of both vocal folds together with the anterior commissure and the anterior part of the thyroid cartilage plates and the subglottic region contained between them.

$>$ partial frontal-lateral laryngectomy - similar to frontal laryngectomy (also modo Leroux-Robert). It consists in the removal of the vocal fold with the anterior commissure and of the adjacent segment of the opposite vocal fold and a fragment of the thyroid cartilage plate with the adjacent fragment of the subglottic region.

$>$ hemilaryngectomy - vertical removal half of the larynx. The surgeon removes part of the thyroid cartilage, including the underlying vocal cord or cords. A very minimal procedure might only remove most of one side of the thyroid cartilage with the underlying soft tissue but not touch the arytenoid cartilage.

$>\quad$ partial supraglottic horizontal laryngectomy - performed by the Alonso method involves removing the upper floor of the larynx to the level of the larynx pockets. Voice folds and arytenoid cartilages are not subject to resection.

Application: cancers located on the epiglottis and vestibular fold.

Due to many types of laryngectomy, laryngectomies also stand out due to the dissection plane - horizontal laryngectomy and vertical laryngectomy. Horizontal laryngectomy is divided into supraglottic laryngectomy - when the vestibule of the larynx is affected by the tumor, glottic laryngectomy - (modo Calearo) - when both vocal folds and anterior commissure are involved and supracricoid laryngectomy - when the upper or middle floor of the larynx is occupied. 


\subsubsection{Total laryngectomy}

Total laryngectomy is the operation where the larynx is finally removed and the airway is disconnected. Respiration being performed through a stoma creating from bringing the trachea to the skin in the lower, cervical area. This provides a total and constant separation of the superior and inferior part of the airway which cause voice and smell loss (8). Total laryngectomy consists of eleven steps (9):

1. Incision and position of stoma

2. Retraction of the SCM (lac. m. sternocleidomastoideus) and division of omohyoid muscle

3. Division of the infrahyoid muscles

4. Repeat steps 2 and 3 on the contralateral side

5. Preservation of one thyroid lobe

6. Separating the constrictor from the thyroid cartilage

7. Releasing the hyoid bone superiorly

8. Division of the trachea

9a. Entry into the pharynx from above

9 b. Entry into the pharynx from below

10. Closure of pharynx

11. Fasion the stoma

Total laryngectomy is addressed mainly to the patients with advanced malignant tumor, posttraumatic laryngeal stenosis, transglottic cancer, cancer recurrence after radiation therapy and failure after partial laryngectomy. Contraindications to laryngectomy is carotid artery involvement, severe diseases and bad condition of patient (8). This procedure can cause many type of complications. The most frequent (29\%) is pharyngocutaneous fistula which is characterized by an abnormal connection between the pharynx and the trachea or skin and is complicated by longer hospitalization, increases morbidity and high level of discomfort. Various factors have been associated with an increased risk of experiencing this type of complication. These factors include anaemia, hypoalbuminaemia, poor nutrition, hepatic and renal dysfunction, preoperative tracheostomy, smoking, alcohol, age, chronic obstructive pulmonary disease and localization and stage of cancer. However, the installation of a freeflap has been shown to significantly reduce the risks of PCF (10). Other complications such as wound infections, recurrence, necrosis, bleeding, hematoma, pharyngeal and stomal stenosis, and dysphagia have also been reported in fewer cases.

\subsubsection{Life after laryngectomy}

The loss of voice and of normal and efficient verbal communication is a negative consequence associated with this type of surgery and can have significant impacts on the quality of life. The main modalities are the electrolarynx, the tracheoesophageal puncture and esophageal speech. All of them have limitations and new technologies may offer modern and personalized alternatives via silent speech. Surface electromyographic (sEMG) signals of articulatory muscles were recorded from the face and neck of a patient who was articulating words silently. These signals were then used for automatic speech recognition via machine learning. Sensor placement was tailored to the patient's anatomy after radioteraphy and surgical treatment (11). Another problem may be hypothyroidism caused by surgery and postoperative radiotherapy, which requires the use of thyroid hormones through whole life. 
Permanent tracheostomy requires proper care and skills to be able to handle the stoma, tracheal tube and another problems and medical aparatures. We can't forget about patient's psyche because this type of patients have been found to be more prone to depression and anxiety. This may be due to the above problems and dysphagia, hiposmia/anosmia and restrictive diet.

\title{
3. Summary
}

The laryngeal cancer can affect all levels of the larynx and give different symptoms depending on location and TNM. The most common method of treatment is laryngectomy, which has different varieties - two main types are partial laryngectomy and total laryngectomy. There is another one subtype which deserves special attention microlaryngectomy. The most common complication after laryngectomy is pharyngocutaneous fistula. Patients' quality of life decreases significantly after surgery, especially due to total laryngectomy (loss of voice and frequent dysphagia). Also postoperative radiotherapy is an important element of treatment. Radiotherapy can also be used as an independent method when TNM is low.

\section{References}

\author{
1. Bochenek A, Reicher M. Anatomia człowieka t.2 Warszawa PZWL 2008:345-359. \\ 2. Becker W, Naumann HH, Pfaltz CR. Choroby uszu, nosa i gardła BEL CORP Warszawa \\ 1999:422-424. \\ 3. http://onkologia.org.pl/rak-krtani/ (dostęp: 2020.04.02). \\ 4. https://www.cancer.gov/types/head-and-neck/patient/adult/laryngeal-treatment-pdq (dostęp: \\ 2020.04.05).
}

5. https://www.cancerresearchuk.org/about-cancer/laryngeal-cancer/stages-typesgrades/stages/TNM-staging (dostęp: 2020.04.02).

6. Dabrundashvili Z, Bakhtadze L, Bakhturidze D, Dabrundashvili N. Treatment and prevention of metastasis to the pre- and paratracheal lymph nodes in cancer of the larynx with spread to the subglottic sectio. Georgian med news. 2019 nov;(296). PubMed PMID: 31889697.

7. Kierzek A. Julian Kosiński (1833-1914) - chirurg szczególnie zasłużony dla rozwoju otolaryngologii. Jego dokonania w leczeniu chorób krtani. Otolaryngologia Polska 2008, LXII, 2:218.

8. Ceachir O, Hainarosie R, Zainea V. Total laryngectomy - Past, Present, Future. Maedica 2014 Jun; 9(2). Pubmed PMID: 25705281.

9. Vlantis AC. Total laryngectomy. CU Medicine Hong Kong (07): 59-67.

http://www.ent.cuhk.edu.hk/images/publication/head-and-neck-dissection-and-reconstructionmanual/07_TOTAL-LARYNGECTOMY.pdf (dostęp: 2020.02.07)

10. Hasan Z, Dwivedi RC, Gunaratne DA, et al. Systematic review and meta-analysis of the complications of salvage total laryngectomy. European Journal of Surgical Oncology (EJSO). 43 (1) (2017-05-27). PubMed PMID: 27265037.

11. Rameau A. Pilot study for a novel and personalized voice restoration device for patients with laryngectomy. Head Neck. 2020 May; 42(5): 839-845. PubMed PMID: 31876090. 